\title{
Kinetics of Crystallization in Multicomponent Systems: I. Binary Mixtures of $n$-Paraffins
}

\author{
John I. Lauritzen, Jr., Elio Passaglia, and E. A. DiMarzio \\ Institute for Materials Research, National Bureau of Standards, Washington, D. C. 20234
}

(April 19, 1967)

\begin{abstract}
The kinetics of crystallization of a binary mixture of $n$-paraffins is treated using a theory for the rate of growth of chains in multicomponent systems. The kinetic chain is considered to be a strip of crystalline material composed of molecules of both components that is growing on a substrate of uniform thickness. This substrate is considered to be a close-packed surface step. Using the binary mixture of $\mathrm{C}_{24} \mathrm{H}_{50}$ and $\mathrm{C}_{26} \mathrm{H}_{54}$ as an example, the calculations are carried out by an iterative procedure so that the mean thickness of the strip equals that of the substrate. This procedure necessitates modification of the rate constants that would be used without this requirement. The rate of growth of the strips (total flux) is calculated as well as their composition over an appropriate range of temperature and a complete range of liquid compositions. The pair distribution in the strips is also determined. The substrate is assumed to be unkinked in the calculations, whereas the substrate is probably highly kinked in the actual system. For this reason the calculated total flux is probably not representative of the actual crystal growth rate in a binary mixture of $\mathrm{C}_{24} \mathrm{H}_{50}$ and $\mathrm{C}_{26} \mathrm{H}_{54}$. It is believed that the calculated compositions are reasonably accurate. For a binary mixture of longer $n$-paraffins (e.g., $\mathrm{C}_{50} \mathrm{H}_{102}$ $\mathrm{C}_{52} \mathrm{H}_{106}$ ) the substrate should have many fewer kinks, and the total flux calculated by this theory will be more closely related to actual crystal growth rate studies. However, no data exist for such systems.
\end{abstract}

Key Words: Crystal growth, multicomponent systems, kinetics, n-paraffins, phase diagrams, pair distributions.

\section{Introduction}

In a recent paper (subsequently abbreviated LDP) $[1]^{1}$ we presented a theory by which the rate of growth and composition of a chain growing in a multicomponent system may be calculated. There are several kinetic problems which may be treated by the method developed in LDP. In particular the chain may be regarded as a layer (or strip) of atoms or molecules at a step on a growing crystal surface, the beginning and end of the chain, or strip, being kinks. The initiation and growth of such strips is one of the processes operative in crystal growth. In fact, in theories of polymer crystallization $[2,3,4]$ it is generally considered that formation of such strips is the rate controlling step in the growth of polymer crystals, and hence the calculation of their rate of growth amounts to the calculation of the rate of growth of these crystals. In polymer crystals the growing step (or lamellar edge) is not expected to be very highly kinked, and this approach appears valid. We treat this problem in the following paper. In atomic systems, the situation is more complicated [5]. The ratecontrolling step is almost certainly not the initiation and growth of these strips, since the step is highly

${ }^{1}$ Figures in brackets indicate the literature references at the end of this paper. kinked [5], and the problem of the calculation of the rate of growth of a crystal becomes essentially a diffusion problem. In systems of intermediate complexity, such as those treated here, the relevance of the kinetics of formation of these strips to the problem of crystal growth is somewhat harder to assess. However, in multicomponent systems, such as we are treating, it seems relatively clear that the elementary processes we treat in calculating the rate of growth and composition of these chains must be important in determining the composition of the crystal, for it must in large measure be the neighbor interaction energies that determine the composition. We thus present an application of the LDP theory to the problem of crystal growth of binary mixtures of $n$-paraffins. The pure normal paraffins are known to grow, at least from solution, by a screw-dislocation mechanism [6], and hence a surface step is present. It is expected that the composition of the chains calculated by this method will be a more accurate representation of the composition of the crystal than the calculated kinetics will be of the rate of crystal growth. However, it is expected that as the length of paraffin molecule increases, the calculated kinetics become a more adequate representation of the rate of crystal growth, for the growing step becomes less and less kinked. 
Although concerned with a specific system, our aim will be less to account for the behavior of this specific system than to illustrate the general behavior of binary systems insofar as this can be done by the LDP theory. Considerable accurate data on the phasediagrams of $n$-paraffin systems are available $[7,8]$ although, to our knowledge, no data are available on the kinetics of crystal growth in such mixtures. The homogeneous nucleation rate has been studied in pure $n$-paraffins [9], but not the crystal growth rate. However, many of the energy considerations relevant to crystal growth are well known [10]. The solidphase behavior of the $n$-paraffins is also well known [10], and, since the LDP theory is applicable only to solid solutions, we will concentrate on a system in which solid-solutions are found at all concentrations. In particular, we will be concerned with the solidification to the hexagonal rotator phase in the system $\mathrm{C}_{24} \mathrm{H}_{50}-\mathrm{C}_{26} \mathrm{H}_{54}$, which meets these requirements. We will ignore any solid-solid phase transitions, and our task will be to calculate the rate of growth of the strips discussed above and their composition as a function of temperature and liquid composition. In what follows we use the words "chain" and "strip" interchangeably.

In order to describe the method of calculation, we will have to describe those aspects of the LDP theory necessary to make the subsequent exposition clear. The LDP theory begins by assuming only nearest neighbor interactions, with rate constants $\alpha^{i j}$ and $\beta^{i j}$, for, respectively, adding species $j$ to a chain ending in species $i$, and removing species $j$ from a chain whose terminal elements are originally $i$ and $j$. These rate constants are assumed to be independent of the length of the chain. However, the rate constants for the initiation of the chain (i.e., the first step in the chain) are different, and are called $\alpha_{0}^{j}$ and $\beta_{1}^{j}$. That is, $\alpha_{0}^{j}$ gives the rate of initiation of new chains from the melt or solution, and $\beta_{1}^{j}$ gives the rate of dissolution of this first step back to melt or solution.

Although there is no limit to the number of components or order of distribution which may be handled by the LDP theory, in this paper we will be concerned only with binary systems and pair distributions. Thus, we define $N_{v}^{j}$ and $\bar{P}_{\nu}^{i j}$ as being, respectively, the number of chains in the system $\nu$ units long with species $j$ in the $\nu$ th position, and $i$ and $j$ in $(\nu-1)$ th and $\nu$ th positions. In this application, of course, $i$ and $j$ can take on only the values 1 and 2 , with 1 representing $\mathrm{C}_{26} \mathrm{H}_{54}$ and 2 representing $\mathrm{C}_{24} \mathrm{H}_{50}$. Clearly, we must have

$$
N_{\nu}^{j}=\sum_{i=1}^{2} P_{\nu}^{i j}
$$

and

$$
N_{\nu}^{T}=\sum_{j} N_{\nu}^{j}=\sum_{i, j} P_{\nu}^{i j}
$$

where $N_{v}^{T}$ is the total number of chains $\nu$ units long. We call these quantities, $N_{\nu}^{j}$ and $P_{\nu}^{i j}$, "occupation num- bers." Kinetic equations for the growth of chains may be formulated [1] in terms of these and more complex quantities. These may be solved at steady state under certain fairly broad conditions. For this purpose we define fluxes $S_{\nu}^{j}$ and $S_{\nu}^{i j}$ as being the net rate of growth of chains (at steady state) from $\nu-1$ to $\nu$ units by, respectively, adding species $j$ to any chain, and to a chain whose terminal $(\nu-1)$ th element is $i$. We obviously have

$$
S_{T}=\sum_{j} S_{\nu}^{j}=\sum_{i, j} S_{\nu}^{i j}
$$

where $S_{T}$ is the total flux. Thus, we may consider that the flux $S_{\nu}^{j}$ is associated with the occupation number $N_{\nu}^{j}$, and the flux $S_{\nu}^{i j}$ with $P_{\nu}^{i j}$.

Under certain and quite unrestrictive conditions on the rate constants, it is shown by LDP that the ratio of flux to its associated occupation number is independent of chain length (for sufficiently long chains) and dependent only on the terminal species in the chain. That is,

$$
\frac{S_{v}^{j}}{N_{\nu}^{j}}=\underset{P_{\nu}^{i j}}{S_{\nu}^{i j}}=\lambda^{j}
$$

where the ratio is denoted $\lambda^{j}$. This in turn is given by the equations

$$
\lambda^{j}=\sum_{i} \frac{\alpha^{j i} \lambda^{i}}{\beta^{j i}+\lambda^{i}}
$$

Moreover, it is also shown in LDP that

$$
N_{\nu}^{j}=\sum_{i} \frac{\alpha^{i j} N_{\nu-1}^{i}}{\beta^{i j}+\lambda^{j}}
$$

For sufficiently large $\nu$, this recursion relation is shown to become [1]

$$
N^{j}=\sum_{i} \frac{\alpha^{i j} N^{i}}{\beta^{i j}+\lambda^{j}} .
$$

Thus for sufficiently long chains, the occupation numbers become independent of chain length, and because of eq (4) the flux also does. "Sufficiently long" in this context is of the order of ten, and thus if the average number of lattice positions between kinks is of this order, this equation will be valid.

Equation (6) is a recursion relation for the $N_{\nu}$. When combined with the equation for the first step in the chain

$$
N_{1}^{j}=\frac{\alpha_{0}^{j} N_{0}^{j}}{\beta_{1}^{j}+\lambda^{j}}
$$

it is entirely possible to build up the chain on a highspeed computer and thus get the initial $\nu$-dependent 
solution as well as the $\nu$-independent limit. (In eq (8) $\alpha_{0}^{j} N_{0}^{j}$ is the rate of initiation of new chains from the melt or solution.)

Now, having the $\nu$ independent limit of $N^{j}$ either by direct solution of eq (7) or iteration of eq (8), the flux is easily obtainable from eq (4):

$$
S^{j}=\lambda^{j} N^{j}
$$

and

$$
S_{T}=\sum_{j} \lambda^{j} N^{j}
$$

It is shown by LDP that the total flux is conserved. Hence, we also have

$$
S_{T}=\sum_{j} \lambda^{j} N_{1}^{j}=\sum_{j} \frac{\alpha_{0}^{j} N_{0}^{j} \lambda^{j}}{\beta_{1}^{j}+\lambda^{j}}
$$

In general we will not know the exact numerical value of $\alpha_{0}^{j} N_{0}^{j}$ or $\beta_{1}^{j}$ and hence will not be able to predict the magnitude of the flux. We hope, however, to be able to predict correctly its dependence on temperature.

Now, the fraction of the $j$ th species in the chain, $f_{j}$, is given by

$$
f_{j}=\frac{S^{j}}{S_{T}}=\frac{\lambda^{j} N^{j}}{\sum_{j} \lambda^{j} N^{j}}
$$

and is thus easily obtained. The pair distributions are also easily obtained. It is shown by LDP that

$$
P_{\nu+1}^{i j}=\frac{\alpha^{i j} N_{\nu}^{i}}{\beta^{i j}+\lambda^{j}}
$$

and for chains that are sufficiently long that the occupation numbers become independent of $\nu$,

$$
P i j=\frac{\alpha^{i j} N^{i}}{\beta^{i j}+\lambda^{j}}
$$

Now, clearly the fraction $f_{i j}$ of $i, j$ pairs in the chain is given by

$$
f_{i j}=\frac{S^{i j}}{S_{T}}=\frac{\lambda^{j} P^{i j}}{S_{T}}=\frac{1}{S_{T}} \frac{\alpha^{i j} \lambda^{j} N^{i}}{\beta^{i j}+\lambda^{j}} .
$$

The steps in the solution are as follows: (a) Having the rate constants $\alpha^{i j}$ and $\beta^{i j}$, compute the $\lambda^{j}$ from eq (5). (b) Having the $\lambda^{j}$, compute the $N_{i, j}^{j}$ by iteration of eq (6) beginning with eq (8) until the $N_{i}^{j}$ arrive at a constant value $N^{j}$. (c) Compute $S^{j}$ by eq (9), and $S_{T}$ by eq (10). (d) Compute the composition of the chain by eq (12) and the pair distributions by eqs (14) and (15). The actual methods used for these computations will be discussed in greater detail below.

\section{The Rate Constants}

\subsection{Preliminary Considerations}

It is shown by LDP that the ratio of the rate constants is given by

$$
\frac{\alpha^{i j}}{\beta^{i j}}=\exp \left[\left(\mu_{j}-e_{i j}\right) / k T\right]
$$

and

$$
\frac{\alpha_{0}^{j}}{\beta_{1}^{j}}=\exp \left[\left(\mu_{j}-\epsilon_{j}^{0}\right) / k T\right]
$$

where $\mu_{j}$ is the chemical potential of the $j$ th species, $\epsilon_{i j}$ is the change in free energy of the growing chain when $j$ is added to a chain ending in species $i$, and $\epsilon_{j}^{0}$ is the change in free energy for the initial element of the chain. There are two things to be noted about eq (16) and (16a). First, they were derived from consideration of an appropriate grand-canonical ensemble, under the principle of detailed balance and as a result kinetic factors such as interfacial transport terms do not appear. Secondly, eqs (16) and (16a) give only the ratio of the forward and backward rate constants in terms of the free energy changes involved in the process. Without more detailed knowledge of the details of the process we do not know how to apportion these free energy changes to the forward and backward rate constants. Rather than try to solve the problem of the detailed process, as has been attempted by Frank and Tosi for chain-folded polymer crystallization [3], we shall take a more empirical approach and solve the problem for various arbitrary apportionments. At equilibrium the results will, of course, be independent of this, and our hope is that this will also be the case under kinetic conditions. As will be seen, our hope is largely fulfilled.

\subsection{Evaluation of $\mu_{j}$ and $\epsilon_{i j}$}

The quantity $\left(\mu_{j}-\epsilon_{i j}\right)$ is the change in free energy of the system in removing species $j$ from the liquid and placing it on the end of a chain whose terminal element is species $i$, and $\left(\mu_{j}-\epsilon_{j}^{0}\right)$ has the analogous interpretation. In order to do this we need both a model of the liquid and a model of the growing crystal. Our philosophy in this is to assume the simplest model possible for both; considering the adequacy of the theory and the state of our knowledge of the kinetics of crystallization in these systems, to assume more detailed models at this state would be presumptuous.

Accordingly, we assume that the liquid is an ideal solution of the two species. For the system considered here $\left(\mathrm{C}_{24} \mathrm{H}_{50}\right.$ and $\left.\mathrm{C}_{26} \mathrm{H}_{54}\right)$ this is not a completely adequate description [11] but neither is it very far wrong. Indeed, for our purposes, it is completely sufficient. We may therefore write the free energy 
$G^{l}(T, P)$ of a liquid mixture of $N_{1}$ molecules of $\mathrm{C}_{26} \mathrm{H}_{54}$ and $N_{2}$ molecules of $\mathrm{C}_{24} \mathrm{H}_{50}$ as

$$
G^{l}(T, P)=N_{1} g_{1}^{l}+N_{2} g_{2}^{l}+N_{1} k T \ln x_{1}+N_{2} k T \ln x_{2}
$$

where $g_{1}^{l}$ and $g_{2}^{l}$ are the free energies per molecule of the pure liquid components at the same temperature and pressure, and $x_{1}$ and $x_{2}$ their mole fractions. We thus have immediately

$$
\begin{aligned}
& \mu_{1}=g_{1}^{l}+k T \ln x_{1} \\
& \mu_{2}=g_{2}^{l}+k T \ln x_{2}
\end{aligned}
$$

To obtain the $\epsilon_{i j}$ and $\epsilon_{0}^{j}$ we adopt a model illustrated schematically in figure 1 . We assume a uniform substrate, or crystal flake, that has already grown with a thickness intermediate between the extended lengths of the molecules, $l_{1}$ and $l_{2}$. (Note that we do not allow any roughness in the substrate. This is discussed more fully below.) Since the solid phase has an hexagonal crystal structure, we assume for simplicity that each molecule in the chain is an hexagonal prism as shown. We now adopt a procedure similar to that used by Price [4] to calculate the energies. Each molecule may be considered to act as a rigid hexagonal prism with a surface free-energy $\sigma$ per unit area. For the difference in length under consideration, this is not a bad assumption. Then, if $g_{j}^{c}$ represents the average free energy per molecule in the

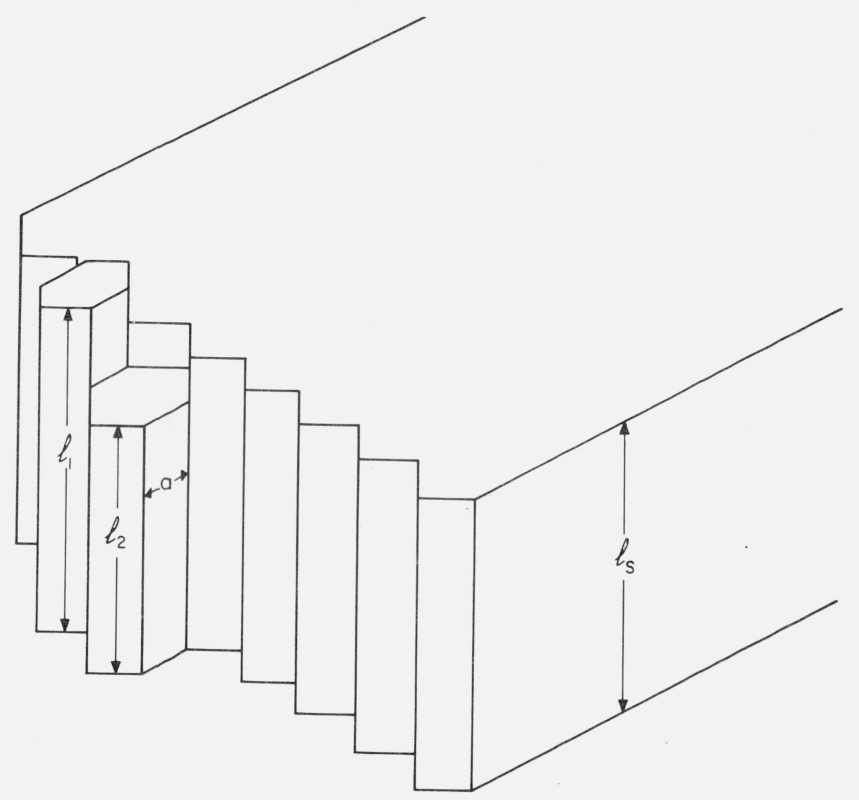

FiguRE 1. Schematic diagram of a model for crystal growth of a binary mixture of $\mathrm{n}$-paraffins.

The growing strip is the kinetic chain of the LDP theory [1]. The lengths of the two paraffin components in extended configuration are $l_{1}$ and $l_{2}$. The cross section of the two components in the crystal are taken to be hexagonal since the solid solution is in the hex agonal rotator phase. The strip is growing on a substrate of uniform thickness, $l_{s}$. pure crystal at the same temperature and pressure, we may write

$$
\epsilon_{i j}=g_{j}^{c}+4 a \sigma U\left(l_{j}-l_{s}\right)+2 a \sigma U\left(l_{j}-l_{i}\right)
$$

where

$$
\begin{aligned}
& U(x)=x \quad x \geqslant 0 \\
& =0 \quad x \leqslant 0
\end{aligned}
$$

and $a$ is the length of the hexagon edge. Note that we have not included a term for the end-surface with free energy $\sigma_{e}$. This merely makes the melting point that of the macroscopic crystal rather than a single lamellar sheet and corresponds to the case of the substrate being a surface step. Hence we obtain for $\mu_{j}-\epsilon_{i j}$

$\mu_{j}-\epsilon_{i j}=\Delta g_{j}-4 a \sigma U\left(l_{j}-l_{s}\right)-2 a \sigma U\left(l_{j}-l_{i}\right)+k T \ln x_{j}$

where $\Delta g_{j}$ is the free energy of fusion (considered as a positive quantity) for species $j$ at the temperature in question. For the first step we obtain

$$
\mu_{j}-\epsilon_{j}^{0}=\Delta g_{j}-4 a \sigma U\left(l_{j}-l_{s}\right)-2 a \sigma l_{j}+k T \ln x_{j} .
$$

Equation (20) indicates that if a molecule with length $l_{j}>l_{s}>l_{i}$ is added to a chain ending in species $i$, corrections to the free energy of fusion must be made because of the newly exposed surface area, but if $l_{j}<l_{s}<l_{i}$, no such corrections need be made. As usual for small supercoolings we assume

$$
\Delta g_{j}=\frac{\Delta h_{j} \Delta T_{j}}{T_{m}^{j}}
$$

where $\Delta h_{j}$ is the heat of fusion and $T_{m}^{j}$ is the melting point appropriate to the species in question and obtain finally

$$
\begin{aligned}
\mu_{j}-\epsilon_{i j}=\frac{\Delta h_{j} \Delta T_{j}}{T_{m}^{j}}- & 4 a \sigma U\left(l_{j}-l_{s}\right) \\
& -2 a \sigma U\left(l_{j}-l_{i}\right)+k T \ln x_{j}
\end{aligned}
$$

where $\Delta T_{j}=T_{m}^{j}-T$, with an analogous expression for the first element.

\subsection{The Effect of the Substrate and the Two- Dimensional Character of the Crystallization Problem}

If we were interested in the strictly one-dimensional problem of the rate of growth and composition of a strip growing on a substrate of fixed length, then the free energies given by eq (20) and the ratios of rate constants calculated from them by eq (16) would be completely appropriate. It would be necessary only to carry out the computations as outlined at the end of the Introduction. This would lead to correct results 
for a one-dimensional problem, for the LDP theory, within the limits of the assumptions, is rigorously correct.

Crystallization, however, presents a problem of a two-dimensional character. What is required as a minimum is that the strip have the same composition as the substrate. In this particular case, this is the same as requiring that the average thickness of the growing strip, defined as

$$
\bar{l}=f_{1} l_{1}+f_{2} l_{2}
$$

be the same as the average thickness of the substrate.

It would appear that this problem could easily be solved by this procedure: (a) Choose a substrate of some convenient thickness. (b) Calculate, by the methods outlined, the composition of a strip growing on this substrate. Its average thickness will in general be different from that of the substrate. (c) Use this strip as a substrate for another strip. (d) Continue this process until the average thickness of the grown strip is the same as that of the substrate. This procedure clearly gives a first and reasonable approximation to the true state of affairs in a growing crystal.

If this is done with the free-energies given by eq (20) and the rate constants calculated from them by eq (16), no matter how the energies are apportioned between the forward and backward reactions, results which are incorrect and aphysical are obtained. If, for example, a phase diagram is calculated by this formalism at equilibrium (total flux, $S_{T}=0$ ), a minimum in the liquidus-composition curve occurs at a different composition from that in the solidus-composition curve. While such a situation could be conceived off equilibrium $\left(S_{T}>0\right)$ it is clearly impossible at equilibrium. This situation does not occur if the computations are carried out at fixed substrate thickness for all compositions of the liquid, but is caused by the varying thickness of the substrate and the resulting two dimensional character of the problem. We must, accordingly, modify the procedure in some manner.

One way of proceeding would be to construct a two-dimensional kinetic theory. This would obviously be a formidable problem, and rather than attack it with very small probability of success, we attempt a more tractable problem. We seek to modify the rateconstant ratio (eq 16) so that at least at equilibrium $\left(S_{T}=0\right)$ physical results are obtained. We then assume that the same form of this ratio will be appropriate under kinetic conditions $\left(S_{T}>0\right)$.

The method used for this is outlined here and developed more fully in the appendix. An ensemble of strips of various lengths and various average thicknesses growing on substrates of various thicknesses is considered. The system is considered to have molecules of two lengths, $l_{1}$ and $l_{2}$, with $l_{1}>l_{2}$. The average thickness of the substrate is assumed to be

$$
l_{s}=l_{2}+f_{s}\left(l_{1}-l_{2}\right)
$$

where $f_{s}$ is the fraction of species 1 in the substrate. Pair or higher order distributions are neglected in the substrate. The average free energy of a strip of length $\nu$ in this ensemble is given by

$$
\begin{aligned}
G_{\nu}=\sum_{j=1}^{2} q_{\nu, 1}^{j}\left[\epsilon_{j}^{0}-\mu_{j}+k T \ln q_{\nu, 1}^{j}\right] & \\
& +\sum_{\eta=2}^{\nu} \sum_{i=1}^{2} \sum_{j=1}^{2} q_{\nu, \eta}^{i j}\left[\epsilon_{i j}-\mu_{j}+k T \ln p_{\nu, \eta}^{i j}\right]
\end{aligned}
$$

where $q_{\nu, 1}^{j}$ is the fraction of strips $\nu$ units long with species $j$ in the first position, $q_{\nu, \eta}^{i j}$ is the fraction of strips $\nu$ units long with species $j$ in the $\eta$ th position and species $i$ in $(\eta-1)$ th position, and $p_{\nu, \eta}^{i j}$ is the probability that if species $i$ is in the $(\eta-1)$ th position in a strip $\nu$ units long, then species $j$ is in the $\eta$ th position. Denoting the number of chains in the ensemble by $N_{T}$, the total free energy is given by

$$
G_{T}=\sum_{\nu=1}^{\nu_{0}} G_{\nu} N_{\nu}+k T \sum_{\nu=0}^{\nu_{0}} N \ln \left(N_{\nu} / N_{T}\right)
$$

The fraction $f$ of species 1 in the strip is clearly given by

$$
f=\frac{\sum_{\nu=1}^{\nu_{0}} N_{\nu} \sum_{\eta=1}^{\nu} q^{1} \nu, \eta}{\sum_{\nu=1}^{\nu_{0}} \nu N_{\nu}}
$$

For given $\mu_{j}, \epsilon_{j}^{0}$, and $\epsilon_{i j}$, the free energy given by eq (25) is a function of $q_{\nu, 1}^{i}, q_{\nu, \eta}^{i j}, N_{\nu}, f_{s}$ and the fraction $f$ of species 1 in the strip.

By variational methods using Lagrange multipliers, a minimum in this free-energy may be sought with respect to these variables, and this will give their values at equilibrium. This analysis is carried out in the appendix.

It is shown that the proper rate constant ratios to obtain the correct distribution at equilibrium are given by

$$
\begin{aligned}
& \frac{\alpha_{0}^{j}}{\beta_{1}^{j}}=x_{j} \exp \left[\Delta h_{j} \Delta T_{j} / T_{m}^{j} k T-2 a \sigma l_{j} / k T\right. \\
& \left.\quad-2 \zeta\left(1-2 f_{s}+f_{s} \omega\right) \delta_{1, j}-2 \zeta f_{s} \omega \delta_{2, j}\right] \\
& \begin{aligned}
\frac{\alpha^{i j}}{\beta^{i, j}}= & x_{j} \exp \left[\Delta h_{j} \Delta T_{j} / T_{m p}^{j} k T-\delta U(j-i)\right. \\
& \left.-2 \zeta\left(1-2 f_{s}+f_{s} \omega\right) \delta_{1, j}-2 \zeta f_{s} \omega \delta_{2, j}\right]
\end{aligned}
\end{aligned}
$$


where the quantities have the following meaning:

$$
\begin{gathered}
\zeta=2 a \sigma\left(l_{1}-l_{2}\right) / k T \\
2 \omega-1=\frac{\xi-x_{2}}{\left[\left(\xi-x_{2}\right)^{2}+\xi x_{2} \exp (-\zeta)\right]^{1 / 2}}
\end{gathered}
$$$$
\xi=x_{1} \exp \left[\Delta h_{1} \Delta T_{1} / T_{m}^{1} k T\right.
$$

$$
\left.-\Delta h_{2} \Delta T_{2} / T_{m}^{2} k T-2 \zeta\left(1-2 f_{s}\right)\right]
$$

and $\delta_{i, j}$ is the Kronecker delta.

At equilibrium we know that $f_{s}=f$. Moreover, as shown in the appendix, $f_{s}=\omega$, and under these conditions physical solutions to the problem are obtained. Hence, without further justification we choose the $\alpha^{i j} / \beta^{i j}$ given by eqs (27) and (28) to be the proper rate constant ratios.

\subsection{The Apportionment of the Energy}

As previously mentioned, without detailed knowledge of the microscopic processes involved, it is not possible to make a decision of how to apportion the free energy between the forward and backward reactions. Since we have no such knowledge we adopt a much more empirical approach and carry out calculations for various arbitrary apportionments. Thus, if we let the argument of the exponential in eq (28) be $A$, then we take

$$
\begin{gathered}
\alpha^{i j}=x_{j} \exp (\varphi A) \\
\beta^{i j}=\exp [-(1-\varphi) A]
\end{gathered}
$$

and carry out calculations for $\varphi=0,1 / 2$ and 1. It will be noticed that the concentration term $x_{j}$ is placed only in the forward reaction. This seems proper in that the probability of adding species $j$ to a growing chain ought to be proportional to the "availability" of that species in the liquid.

For the initial step, if we let the argument of the exponent in eq (27) be $A^{\prime}$, we take

$$
\begin{aligned}
& \alpha_{0}^{j}=x_{j} \exp \left(A^{\prime}\right) \\
& \beta_{1}^{j}=1 .
\end{aligned}
$$

That is, for the initial step, we put all the energy into the forward reaction and take the backward reaction to be a constant. This affects only the magnitude of the flux, which, as previously mentioned, we cannot in any case expect to predict exactly.

\subsection{The Transport Term}

Equations (16), (27), and (28) for the rate constant ratios, being the result of equilibrium considera- tions, do not involve any transport (or diffusion) terms. The inclusion of such terms in eqs (29) and (30) poses difficult problems, for in general these terms are not known. If these transport terms depend upon both the species at the end of the chain and the species coming on or going off (i.e., depend upon both $i$ and $j$ ) then their omission can lead to serious problems. Thus, if $\alpha^{\prime i j}$ and $\beta^{\prime i j}$ are the rate constants including transport terms, the most general relation to the $\alpha^{i j}$ and $\beta^{i j}$ are

$$
\alpha^{\prime i j}=a^{i j} \alpha^{i j}
$$

and

$$
\beta^{\prime i j}=b^{i j} \beta^{i j}
$$

where $a^{i j}$ and $b^{i j}$ represent the transport terms. At equilibrium, of course, we must have $a^{i j} / b^{i j}=1$. These new rate constants determine new $\lambda^{j}$ by eq (5) and hence new compositions and fluxes. These coefficients are, of course, unknown, and indeed in such theories as this are usually left as undetermined parameters.

In the present case however, the similarity of the two species in question is such that a reasonable approximation to the true state of affairs may be obtained with a much more restrictive condition than eq (31). In the particular case under consideration here we could adopt these relations

$$
\begin{aligned}
& \alpha^{\prime i j}=a \alpha^{i j} \\
& \beta^{\prime i j}=b \beta^{i j} .
\end{aligned}
$$

Now, as long as $S_{T}=0$, we know the ratio $a / b$ is unity from eq (16), and we note that this statement is not restricted to the melting temperature. At the melting point, of course, $S_{T}=0$, and $a=b$. For finite currents not too far below the melting point this latter equality is expected to be an excellent approximation, if not indeed a rigorous fact.

Thus, taking $a=b$, and calculating the $\lambda^{\prime i}$ corresponding to $\alpha^{\prime i j}$ and $\beta^{\prime i j}$ we have

$$
\lambda^{\prime i}=\sum_{j} \frac{a \alpha^{i j} \lambda^{\prime j}}{a \beta^{i j}+\lambda^{\prime j}}
$$

whence

$$
\frac{\lambda^{\prime i}}{a}=\sum_{j} \frac{\alpha^{i j} \lambda^{\prime j} / a}{\beta^{i j}+\lambda^{\prime j} / a}
$$

and

$$
\lambda^{\prime i}=a \lambda^{i}
$$

Inspection of eq (6), (12), and (15) shows that the transport term as represented by the quantity $a$ has 
no effect on the occupation numbers and the composition, but inspection of eq (10) shows that the flux calculated without the transport term must be multiplied by the factor $a$ to obtain the flux when the transport term is included. In the calculations carried out here we take $a$ to be unity, for, as previously stated, we cannot expect to get the true magnitude of flux in any case. In addition, however, the temperature dependence of the flux we calculate will be slightly wrong, and should be multiplied by an Arrhenius type term. For the undercoolings involved in this work (at most several degrees) this causes a negligible error in the temperature dependence.

\section{Method of Calculation}

The energies and molecular lengths chosen for the calculation were taken from a paper by Broadhurst [10]. The lateral molecular dimensions were computed to give the cross-sectional area per molecule given by $V$ and [12]. The data and conditions are summarized in table 1. Of particular importance in the table are the values of surface free energy, $\sigma$, which were chosen to be 3,4 , and 8 ergs $/ \mathrm{cm}^{2}$. This is lower than the $8-12$ ergs $/ \mathrm{cm}^{2}$ obtained from the data of Turnbull and Cormia [9]. Since we are considering the transition from liquid to hexagonal rotator phase we expect $\sigma$ to be lower, and indeed, comparison with the data of Mazee [7] shows that it most probably is.

The calculations were carried out on an IBM 7094 computer as follows.

(a) For a given value of $\varphi$ and a given concentration $x_{1}$ and $x_{2}\left(=1-x_{1}\right)$ in the liquid, a starting value of $f_{s}=x_{1}$ was chosen and rate constants calculated by eqs (29), (30), (27), and (28).

(b) From these rate constants, the $\lambda$ were determined by eq (5). These were solved by an iteration procedure outlined in LDP. Starting with

$$
B_{0}^{i}=\sum_{j} \alpha^{i j} / \beta^{i j}
$$

we calculate successively

$$
B_{p}^{i}=\sum_{j} \frac{\alpha^{i j} B_{p-1}^{j}}{\beta^{i j}+B_{p-1}^{j} .}
$$

TABLE 1. Data and conditions used for calculations

\begin{tabular}{c|c|c|c|c}
\hline \hline Compound & Length, $\AA$ & $\Delta h_{f} / k$ & $T_{m},{ }^{\circ} \mathrm{K}$ & $\begin{array}{c}\text { Concentration } \\
\text { in liquid }\end{array}$ \\
\hline $\mathrm{C}_{24} \mathrm{H}_{50}$ & 32.46 & 6590 & 323.8 & $0-1$ \\
$\mathrm{C}_{26} \mathrm{H}_{54}$ & 35.00 & 7150 & 329.5 & $0-1$ \\
\hline
\end{tabular}

$a=2.795 \AA$

$\sigma / k, \AA^{-2}=2.172,2.896,5.792$

$\varphi=0,1 / 2,1$

Temperature: $315^{\circ} \mathrm{K}$ to melting point. until convergence is obtained. It is shown by LDP that this series converges to $\lambda^{j}$. This is easier than solving eq (5) for the $\lambda^{j}$ directly. Moreover, for $n>2$ such direct solution is usually impossible. Iteration was carried out until $B_{p}^{j}$ was within 1 part in $10^{7}$ of $B_{p-1}^{j}$. Convergence took only a few hundred steps except near the melting point $\left(\lambda^{j}=0, S_{T}=0\right)$ where convergence was slow ( $10^{4}$ steps). The series does not converge for $T$ above the melting point for that particular composition $\left(\lambda<0, S_{T}<0\right)$.

(c) Having the $\lambda^{j}$, the $N_{\nu}^{j}$ were obtained by successive iteration of eq (6) starting with eq (8) until convergence to 1 part in $10^{7}$ was obtained. $N_{0}^{j}$ was chosen to be $10^{6}$.

(d) The quantities $S_{j}, S_{T}, f_{j}$, and $f_{i j}$ were calculated by eqs (9), (10), (12), and (15), respectively.

(e) Having calculated $f_{j}$ we of course have the composition of the grown strip. We now choose $f_{s}=f$ (the fraction of species 1 in the strip) and repeat the procedure, and continue this until the calculated $f$ is equal to $f_{s}$ to one part in $10^{6}$.

The range of parameters given in table 1 takes about five minutes of computation time.

\section{Results}

What we wish to know from the computations are (a) The rate of growth (total flux, $S_{T}$ ) as a function of temperature and liquid composition.

(b) The composition of the crystal, $f$, crystallizing from a liquid of composition $x_{1}$ as a function of $x_{1}$ and temperature.

(c) The pair distributions $f_{11}, f_{12}$, and $f_{22}$.

To achieve these aims, computations were carried out for the values of the parameters listed in table 1 . Calculations were carried out at liquid compositions $x_{1}$ from 0 to 1 by increments of 0.1 For each composition calculations were carried out for all the integer values of temperature from $315{ }^{\circ} \mathrm{K}$ up to the closest integer value to the temperature at which $S_{T}=0$. For each of the quantities of interest we are particularly interested in determining the effect of $\sigma$ and $\varphi$.

\subsection{The Total Flux}

Representative results for the total flux $\left(S_{T}\right)$ are given in figure 2. We have illustrated its behavior with temperature by giving plots of it against temperature for $\sigma=4$ and 8 and $\varphi=0$ and 1 . The curves for the other values of the parameters are very similar.

In all cases the total flux is essentially linear with temperature, but perhaps more striking is the fact that the slope of these curves is almost independent of composition. This means that the total flux is dependent essentially only on the undercooling appropriate to that composition. This is not a totally unexpected result, for the change in crystal free energy with composition (aside from entropy of mixing effects) is small considering the similarity of the 

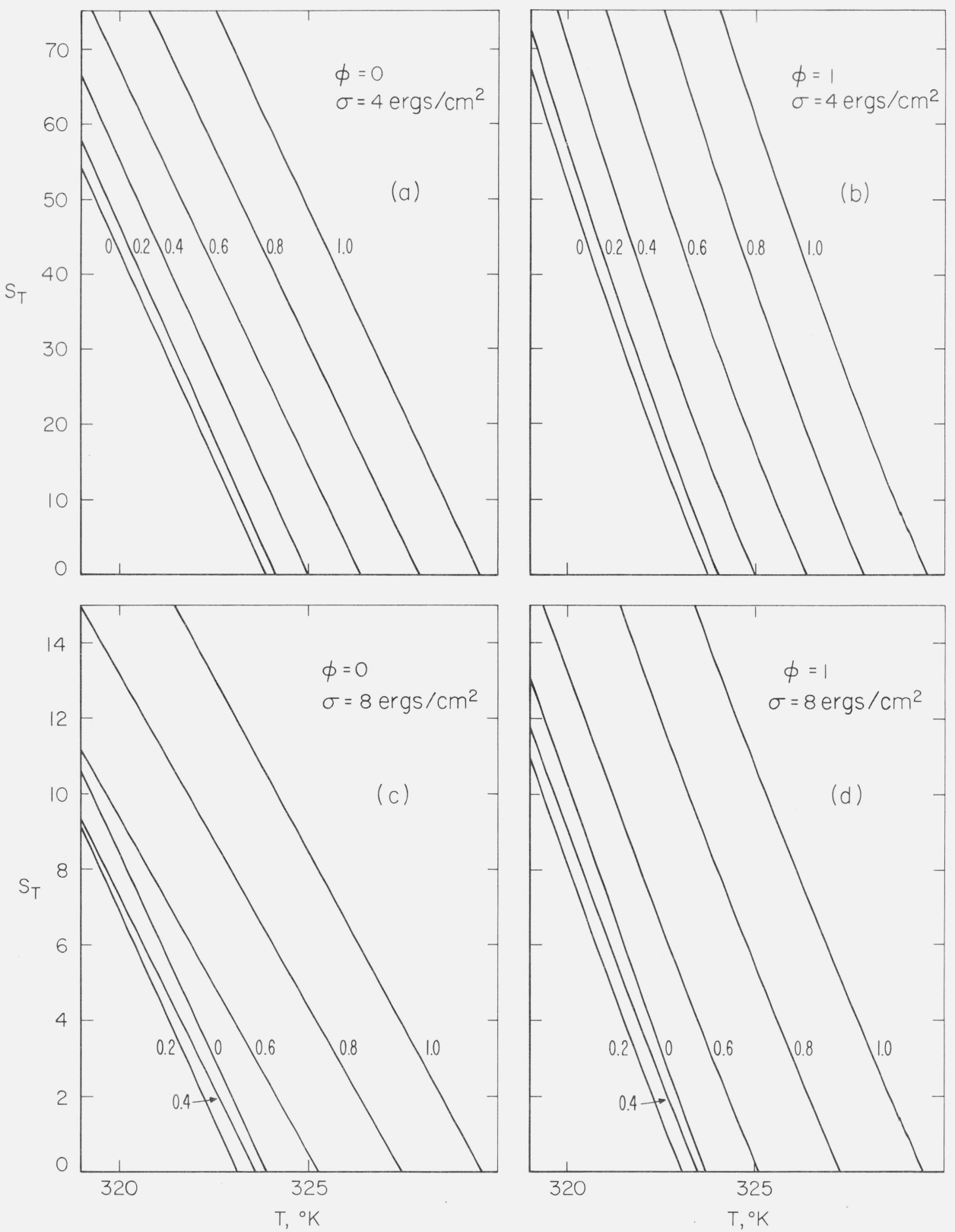

Figure 2. Plots of total flux of crystallization versus temperature in binary mixtures of $\mathrm{C}_{24} \mathrm{H}_{50}$ and $\mathrm{C}_{26} \mathrm{H}_{54}$

Each of the four figures have plots for six values of liquid concentration of $\mathrm{C}_{26} \mathrm{H}_{54}$ ranging from 0 to 1. Figures 2(a) and 2(b) are calculated for $\sigma=4 \mathrm{ergs} / \mathrm{cm}^{2}$ and for $\varphi=0$ and $\varphi=1$, respectively. Figures 2(c) and 2(d) are calculated for $\sigma=8 \mathrm{ergs} / \mathrm{cm}^{2}$ and for $\varphi=0$ and $\varphi=1$, respectively. All plots are quite linear. When $\sigma=4 \mathrm{ergs} / \mathrm{cm}^{2}$ the curves are displaced uniformly to higher temperatures with increasing liquid concentration of $\mathrm{C}_{26} \mathrm{H}_{54}$; when $\sigma=8 \mathrm{ergs} / \mathrm{cm}^{2}$ the curves are first displaced to lower and then to higher temperatures with increasing liquid concentration of $\mathrm{C}_{26} \mathrm{H}_{54}$. This behavior results from the minimum that exists in the liquidus and solidus curves for the higher value of $\sigma$.

dimensions of the molecules. To the extent that these curves can be interpreted as rates of crystallization, they imply that this rate is dependent almost entirely on the undercooling appropriate to that composition.

A detailed analysis of these curves shows that their slopes are related systematically. If the average slope over the illustrated temperature interval is indicated by $m(\varphi, \sigma)$ then the following approximate relations hold:

$$
\frac{m(\varphi, \sigma)}{m(0, \sigma)}=f(\varphi)
$$


approach the temperature axis horizontally for a $\sigma$

$$
\frac{m(\varphi, \sigma)}{m\left(\varphi, \sigma_{0}\right)}=g(\sigma)
$$

where $f(\varphi)$ and $g(\sigma)$ are functions only of $\varphi$ and $\sigma$, respectively. This means that the variation of these slopes with $\varphi$ for any given $\sigma$ is independent of $\sigma$ and the variation with $\sigma$ for any given $\varphi$ is independent of $\varphi$.

As previously discussed under the section on the transport terms, these slopes are not correct, for they do not contain the effect of this term. They should be multiplied by the factor $a$ which is expected to be of the form

$$
a=a_{0} \exp [-\Delta H / k T] .
$$

However, unless the preexponential factor $a_{0}$ and the activation energy $\Delta H$ are inordinately large, this will have only a negligible effect on the slopes over the eight degree range of undercoolings considered.

\subsection{The Crystal Composition}

The composition $f$ of a strip crystallizing from a liquid with composition $x_{1}$ for various values of the parameter $\sigma$ is shown in figures 3 and 4 . Figure 3 is computed for $S_{T}=0$ at all compositions and thus is a true phase diagram. The effect of the parameter $\varphi$ is indistinguishable on the scale of these figures.

Figure 3 indicates that as the surface energy of the material is increased, a minimum in the phase diagram occurs, and the maximum separation between liquidus and solidus increases. Since $\sigma$ is a measure of the interaction energy between the two species in the solid phase, this behavior is normal. Detailed calculations indicate that the liquidus and solidus curves

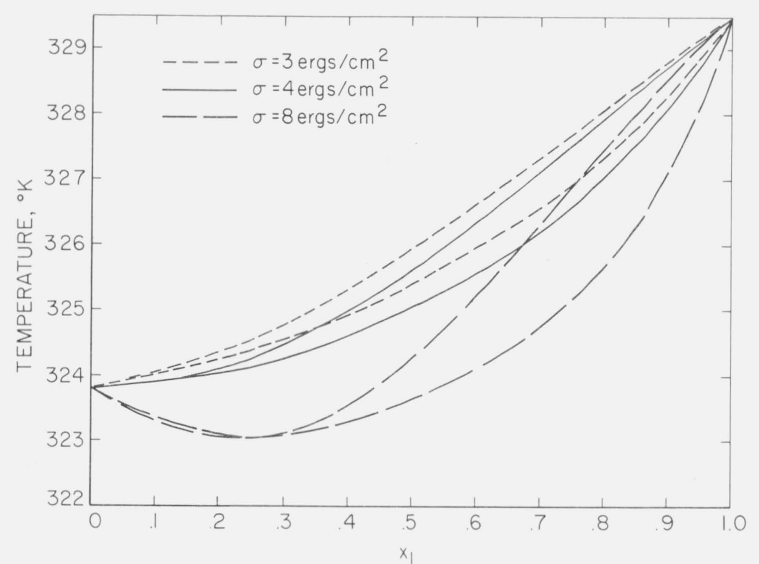

Figure 3. Phase diagrams of the binary mixture of $\mathrm{C}_{24} \mathrm{H}_{50}$ and $\mathrm{C}_{26} \mathrm{H}_{54}$ for three values of $\sigma$.

$x_{1}$ is the molar concentration of $\mathrm{C}_{26} \mathrm{H}_{54}$. This diagram represents the composition of the solid at equilibrium $\left(S_{T}=0\right)$ with a given liquid composition. The solidus curve falls below or touches the liquidus curve. As $\sigma$ increases above $4.018 \mathrm{ergs} / \mathrm{cm}^{2}$ a temperature minimum or touches the liquidus curve.
occurs in the phase diagram. value of $4.018 \mathrm{ergs} / \mathrm{cm}^{2}$. The experimental curves given by Mazee [7] for this system indicate that the liquidus and solidus curves do not have such a horizontal portion, and we conclude that $\sigma$ for the hexagonal rotator phase of the normal paraffins is somewhat less than $4 \mathrm{ergs} / \mathrm{cm}^{2}$. This is to be compared with the value of about $12 \mathrm{ergs} / \mathrm{cm}^{2}$ deduced from the data of Turnbull and Cormia [9] for the nonrotator phases.

Figure 4 looks superficially like the phase diagram, figure 3 , but is not, for it is for kinetic conditions and hence the phase rule, which is derived for equilibrium, cannot be applied to it. This diagram gives the composition of the solid separating from a given liquid composition at constant total flux $S_{T}$ and we call it a "kinetic phase-diagram," and even use the terms "liquidus" and "solidus," even though not entirely appropriately. The constant total flux was chosen by choosing the crystallization temperature for the pure $x_{2}$ case to be $321.0^{\circ} \mathrm{K}$ (undercooling of $2.8{ }^{\circ} \mathrm{C}$ ). The composition of crystal separating from a liquid of composition $x_{1}$ was then computed for this same flux, as well as the temperature at which this occurred. By the remarks made about the behavior of the total flux with temperature and composition, this gives almost a constant amount of undercooling throughout the diagram, but not quite, as is apparent from the $x_{1}=1$ end of the diagram.

The curves of figure 4 are superficially very similar to those of figure 3 , and indeed it would take extremely careful experiments to be able to distinguish between the two. This indicates that even if conditions of exact equilibrium do not obtain when determining phase diagrams for this system, very little error will be made.

A more detailed analysis shows, however, that there are differences between these kinetic phase

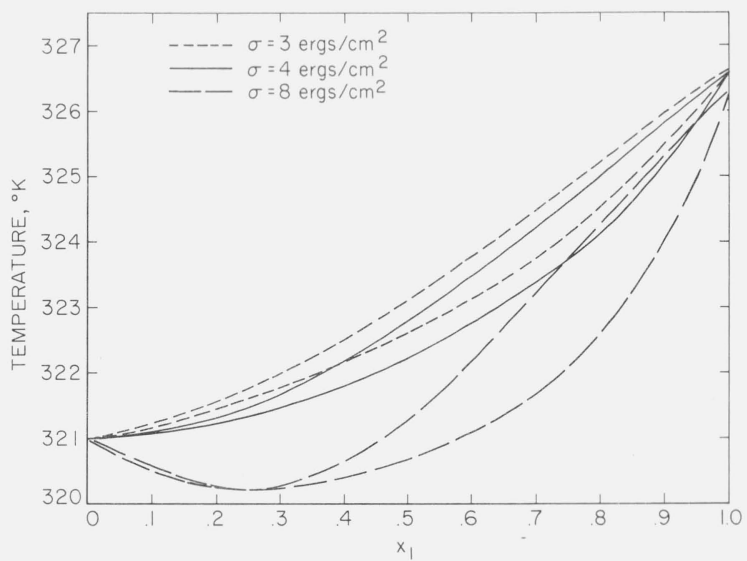

Figure 4. A "Kinetic phase-diagram" of the binary mixture of $\mathrm{C}_{24} \mathrm{H}_{50}$ and $\mathrm{C}_{26} \mathrm{H}_{54}$ for three values of $\sigma$.

$x_{1}$ is the molar concentration of $\mathrm{C}_{26} \mathrm{H}_{5+4}$. This diagram represents the composition of the olid separating from a viven liquid concentration at constant total flux $S_{\text {. }}$. The total flux was chosen by chowsin the crystallization temperature for the case $x_{1}=0$ to be $3210^{\circ} \mathrm{K}$ (undercolin of $2.8^{\circ} \mathrm{K}$. The composition of the erytat sor $x_{1}$ was 2.8 . $\mathrm{K}$. The compesition of the crystal separ ating from a liquid of composition $x_{1}$ was compated for this same flux as well as he tenperature at which this occurred. These curves have almost. but not quite, a constant amount of undercooling. The effect of the parameter $\varphi$ is indistinguishable on the scale of this figure. 
diagrams and the equilibrium phase diagram. The most striking difference is shown in figure 5 which is an enlargement of the region of the minimum for the curve calculated for $\varphi=1$ and $\sigma=8$ for the same total flux as in figure 4 . This figure indicates that the minimum in the temperature-liquidus curve occurs at a different composition from that in the temperature-solidus curve. This could not, of course, occur in an equilibrium phase diagram, but there is nothing to preclude it happening in these kinetic diagrams. To the right of the vertical arrow the composition of the crystallizing solid is higher in species one than that of the liquid $\left(f>x_{1}\right)$ while to the left it is lower $\left(f<x_{1}\right)$. This is denoted by the horizontal arrows. At the point denoted by the vertical arrow the composition of the solid is the same as that of the liquid. This "equal-composition" point, which we denote by $X_{e}$, for an equilibrium phase diagram must, of course, occur at the minimum of the liquidus and solidus curves, but no such restriction occurs here.

It is to be noted that although the minimum in the liquidus and solidus curves occur at different compositions, the temperatures at the minima are the same. For this not to be true would require a double valuedness in the relation between $f$ and $x_{1}$. But our $\alpha^{i j}, \beta^{i j}$, and $\lambda^{j}$ and the quantities calculated directly from them are continuous and single valued. Hence, this double-valuedness cannot exist, and the temperature at the minimum in both the solidus and liquidus curves must be the same. It is also clear on physical grounds, that any point on the liquidus curve must correspond to a point on the solidus curve, and vice versa. Thus the minimum in the two curves must be at the same temperature.

It is interesting to consider the effect of supercooling on the composition $X_{e}$. As the undercooling is increased, $X_{e}$ decreases, and this is illustrated

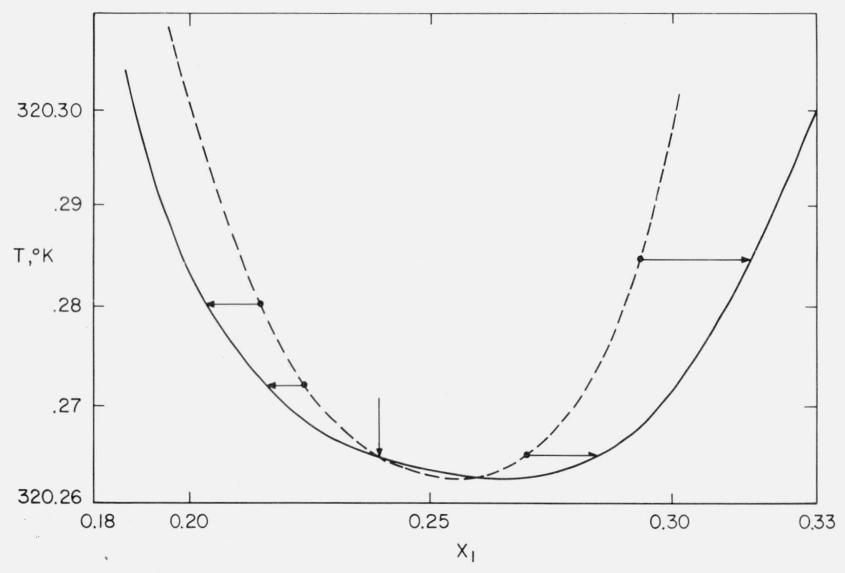

FIGURE 5. An enlargement about the minimum of the "kinetic phase diagram" of figure 4 for $\sigma=8 \mathrm{ergs} / \mathrm{cm}^{2}$ and $\varphi=1$.

Here the minima of the temperature-solidus (solid line) and the temperature-liquidus (dashed line) curves do not occur at the same composition. This could not occur in an equilibrium phase diagram. To the right of the vertical arrow the composition of the crystallizing solid is higher in $\mathrm{C}_{26} \mathrm{H}_{54}$ than that of the liquid, while to the left it is lower. This is indicated by the horizontal arrows. The vertical arrow indicates the "equal composition" point. schematically in figure 6 . In this figure we show the equilibrium phase diagram and one of the family of kinetic phase diagrams. On this figure there are shown three curves intersecting the kinetic phase diagram. These represent schematically the locus of $X_{e}(T)$, the locus of the minimum in the liquidus curve, $X_{m}(T)$, and the locus of points on the liquidus conjugate (i.e., at the same temperature) to $X_{e}$. This last locus we denote by $X_{e}^{c}$. The form of these curves is difficult to develop analytically. This much, however, can be said. The slope of $X_{e}(T)$ and $X_{m}(T)$ at the equilibrium diagram is negative. It does, however, depend upon $\varphi$ and the $\sigma$ involved, and this sign of the slope is not a general rule. The slope at the point where these curves cross the $x_{1}=0$ axis is positive, and also depends upon $\varphi$.

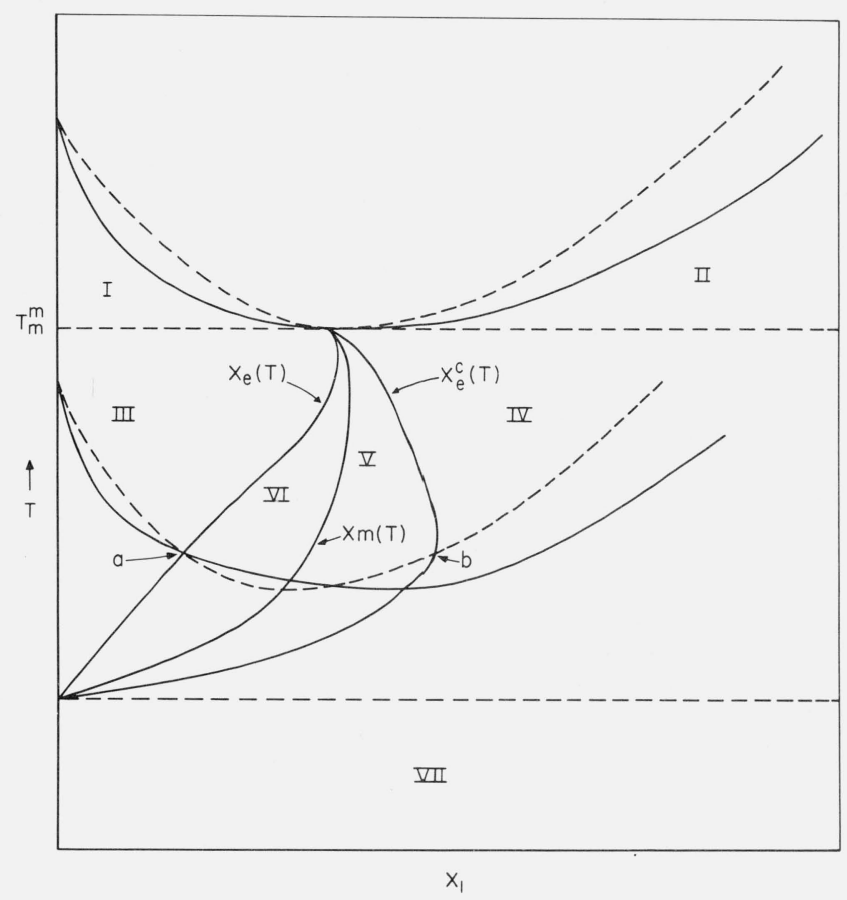

FIGURE 6. Schematic presentation of the equilibrium phase diagram and one of the family of "kinetic phase diagrams" of a binary mixture of n-paraffins.

Three curves intersect the "kinetic phase diagram." These curves represent schematically the locus of the equal composition point, $X_{e}(T)$, the locus of the minimum in the liquidus curve, $X_{m}(T)$, and the locus of the points conjugate to $X_{e}(T)$ (i.e., at the same temperature on the liquidus curve), $X_{e}^{c}(T)$. The temperature minimum of the equilibrium phase diagram is $T_{m}^{m}$. The supercooled area beneath the equilibrium phase diagram can be divided into 7 regions designated by Roman numerals and two points, $\mathbf{a}$ and $\mathbf{b}$. In these 7 regions and at these two points the time dependence of the total flux are qualitatively different.

Moreover, the temperature $\bar{T}$ at which $X_{e}=0$ can be determined analytically. This can be shown to be

$$
\bar{T}=\frac{\Delta h_{1}-\Delta h_{2}-6 a \sigma\left(l_{1}-l_{2}\right)}{\Delta h_{1} / T_{1}-\Delta h_{2} / T_{2}} .
$$

For the parameters for the curves in figures 4 and 5 , $\bar{T}=232.5^{\circ} \mathrm{K}$ which is a completely unattainable under- 
cooling. For $\sigma=4.05 \mathrm{ergs} / \mathrm{cm}^{2}$ and all other parameters the same as for the above curves, $\bar{T}=322.90$ which is an undercooling of only $0.9 \mathrm{deg}$, and hence in principle observable. However, in this case the minimum is very slight. For the system $\mathrm{C}_{30} \mathrm{H}_{62}-\mathrm{C}_{35} \mathrm{H}_{72}$, for which experimental data are available and a minimum exists, $\bar{T}=191{ }^{\circ} \mathrm{K}$, and hence also completely unattainable. We thus conclude that the existence of $\bar{T}$ is a prediction of the LDP theory which is likely to remain academic.

The kinetic phase-diagrams in figures 4 and 5 give the composition of solid crystallizing at a given rate from a given composition of liquid. In other words, in a real system this gives the composition of the very first crystal formed. As time proceeds, however, the composition of the liquid changes, and it is interesting to deduce what happens to the total flux as time proceeds.

The most interesting case is that in which the phase diagram has a minimum, and the behavior in this case can be described with reference to figures 6 and 7. Figure 6 shows the equilibrium phase diagram and one of the family of constant flux kinetic phase diagrams. Those with smaller fluxes are above the illustrated kinetic phase diagram, and those with larger below. It is convenient to divide the temperature-composition space into seven areas, as shown, and two points, $\mathbf{a}$, and $\mathbf{b}$.

What happens to the total flux when a liquid of a given composition is brought to a temperature below the melting point and held at the temperature is shown for each of the seven regions and the two points in figure 7 . The diagrams are almost self explanatory, but we will describe them briefly.

In regions I and II, as the liquid crystallizes, its composition changes such that the point describing it moves into areas where the crystallization rate is lower, finally reaching a point on the equilibrium liquidus curve at which time the flux drops to zero and crystallization ceases.

In regions IV and $\mathrm{V}$, the flux initially decreases, but because the point representing the liquid composition crosses the curve, $X_{m}(T)$, the flux begins to increase again, eventually leveling off where the point reaches the $X_{e}(T)$ curve at, respectively, lower and higher values. If the initial liquid composition and temperatures are such that the initial liquidus point is on the $X_{e}^{c}(T)$ curve (point $b$ ), the flux first drops, but then increases back to its original value
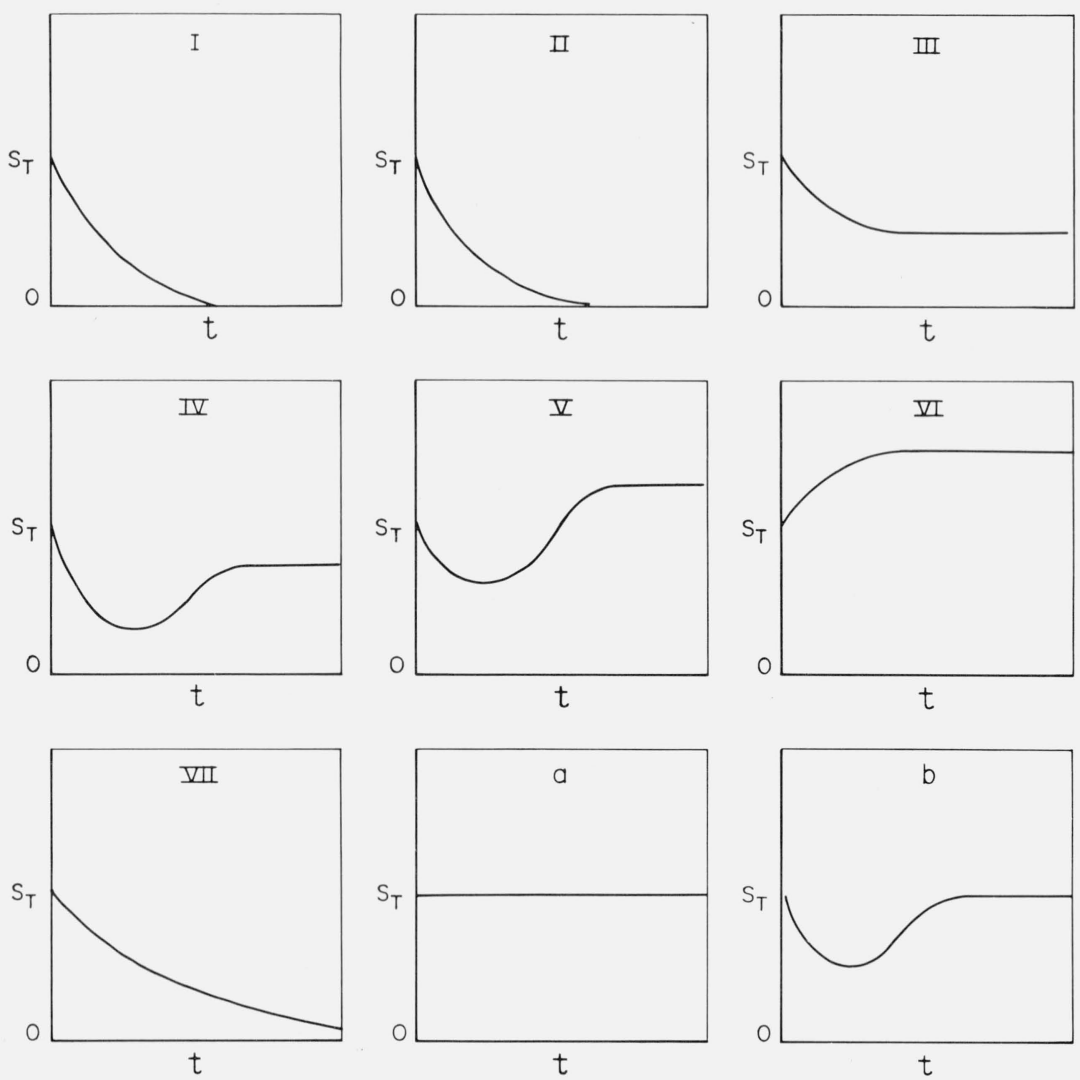

FIGURE 7. Schematic plots of total flux, $\mathrm{S}_{\mathrm{T}}$, versus time for a binary mixture of $\mathrm{n}$-paraffins that is represented in figure 6.

Each plot represents schematically the time dependence of the total flux when crystallization begins in the indicated regions or at the indicated points of figure 6 . In regions I and II crystallization does not proceed to completion. 
when the liquid point reaches $X_{e}(T)$ and remains constant thereafter.

In region VI the flux increases monotonically since all points in this region are to the left of $X_{m}(T)$ eventually becoming constant when the liquid point reaches $X_{e}(T)$. In region III, the flux decreases monotonically until the liquidus point reaches $X_{e}(T)$ after which it becomes constant.

If the initial composition and temperature are such that the liquid point is on the curve $X_{e}(T)$ (point a), the composition and hence the flux are independent of time. This is clearly a generalization of the behavior at the minimum in the equilibrium curve.

In region VII (all points below $\bar{T}$ ) the flux decreases monotonically in time and reaches a constant value only when the species 1 is completely depleted from the liquid.

It should be pointed out again that although this behavior is a direct consequence of the LDP treatment, its experimental verification is likely to be very difficult, and its experimental importance relatively slight. In any real system the curves $X_{e}(T), X_{m}(T)$ and $X_{e}^{c}(T)$ are almost certain to be practically coincident and vertical, and region VII will be unattainable. Thus, regions V, VI, and VII will practically disappear, and the behavior in all attainable regions will be such that the flux will decrease monotonically - to zero if the liquid point is above the minimum melting temperature but below the liquidus curve, and to a constant value if the liquid point is below the minimum melting temperature.

The last question we have to investigate is the occurrence of a eutectic. It is clear from what has been said about the continuous single-valued nature of our $\alpha^{i j}, \beta^{i j}$ and $\lambda^{j}$ with respect to $x_{1}$ and $f$, that we will never obtain a eutectic unless we include its possibility in a more or less ad hoc manner. To do this it would be necessary to investigate the free-energy of the solid phase with respect to composition and determine if in some composition range the free energy of the solid is lower when expressed as a linear combination of two fixed solid compositions in standard ways [13]. For the values of the interaction parameter $(\sigma)$ chosen here, this does not occur and no eutectic is formed. A simple calculation indicates that a value of $\sigma$ greater than approximately $22 \mathrm{ergs} / \mathrm{cm}^{2}$ is necessary before eutectic formation occurs at any reasonable undercooling. This is an unreasonably high value of surface energy for $n$-paraffins.

\section{The Pair Distribution}

As indicated in the introduction the pair distribution is easily calculated by the formalism of LDP. In order best to present typical results, in figure 8 we show plotted against composition $f$ the difference between the observed $f_{12}$ and the random mixing case, where $f_{12}$ is given by $f(1-f)$. These are computed along the solidus line in figure 4 for $\sigma=4$ and $8 \mathrm{ergs} / \mathrm{cm}^{2}$. This curve gives all the possible informa-

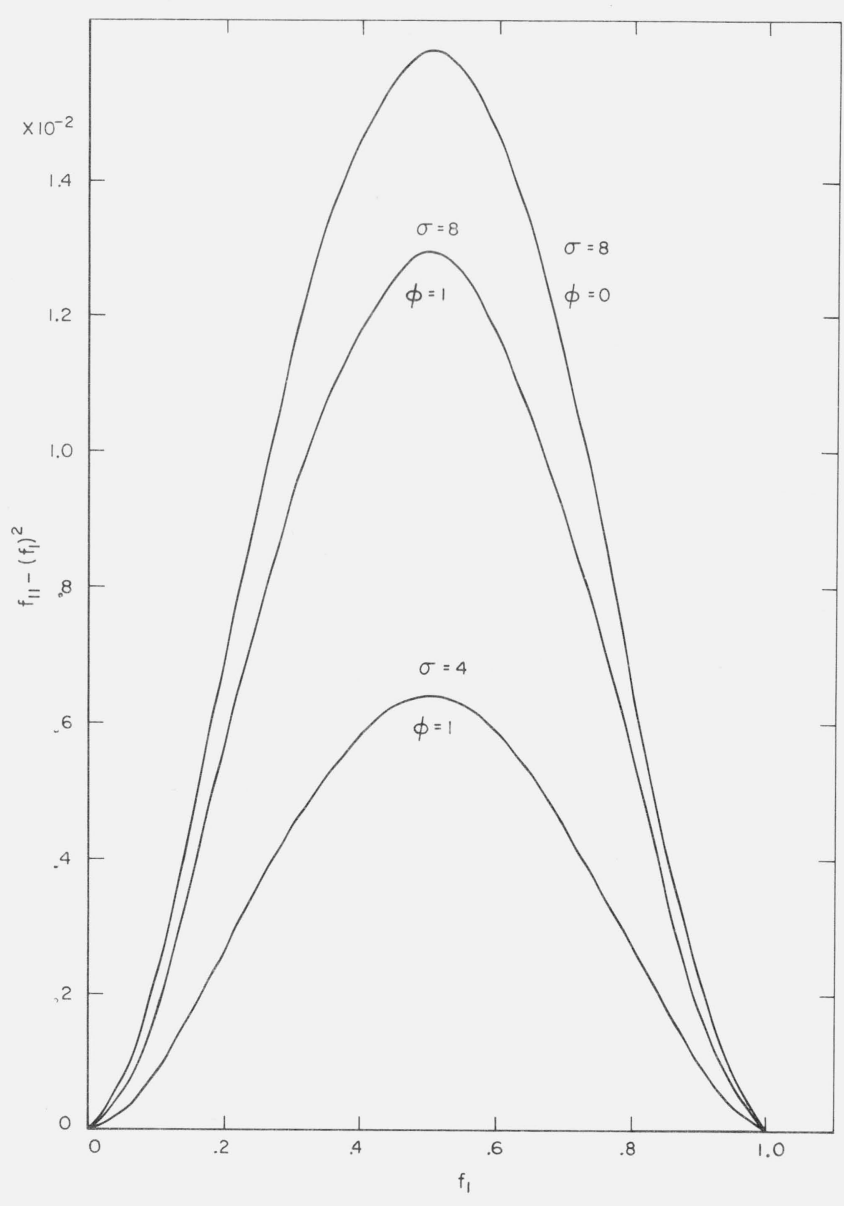

Figure 8. Plot of the difference between the pair distribution $\mathrm{f}_{11}$ and the random mixing pair distribution $\left(\mathrm{f}_{1}\right)^{2}$ versus the singlet distribution $\mathrm{f}_{1}$ for various indicated values of $\sigma$ and $\varphi$.

These curves are calculated along the solidus curve in figure 4 . The deviations from random mixing are very small.

tion about the pair distributions, for the following identities hold:

$$
\begin{aligned}
f_{12} & =f_{21} \\
f(1-f)-f_{12} & =f_{11}-f^{2} \\
f_{11}-f^{2} & =f_{22}-(1-f)^{2} .
\end{aligned}
$$

First to be noticed in figure 8 is the small deviation of $f_{12}$ from the random distribution. In the worst case this amounts to only about 6 percent at the $f=1 / 2$ point. This near equivalence is to be expected from the small magnitude of the interaction energy as expressed by $6 a \sigma\left(l_{1}-l_{2}\right)$ in comparison to the entropy of mixing terms.

The effect of $\sigma$ is such as to increase the deviation from randomness, as is to be expected, and the deviation is essentially proportional to $\sigma$ for a given value of $\varphi$. The latter parameter causes a not inconsiderable effect, but we have no explanation for it.

The curves show an apparent symmetry about the point $f=0.5$. If this symmetry were indeed present, 
it would mean that $f_{12}$ is symmetric about $f=0.5$ since clearly the random case is. However, this symmetry is only apparent. Detailed investigation near $x=0$ and $x=1$ indicate that $f_{12}$ is not symmetric about $f=0.5$. This is what would be expected considering the form of Equation 28, but the point is too recondite to pursue.

\section{Conclusions}

We have shown that by relatively routine calculations using the LDP method, it is possible to calculate the kinetics of growth and composition of new layers growing at a surface step on a growing crystal in a binary mixture when the solid phase forms a solid solution. This procedure is expected to be an adequate representation of one of the processes occurring in crystal growth when the average distance between kinks is of the order of ten or more lattice spacings. If this distance is very large then this calculation would give the kinetics of crystal growth, but it is expected that in the present application the results will be more accurate for the composition. If the calculations were made for a binary mixture of longer hydrocarbons (e.g., $\mathrm{C}_{50} \mathrm{H}_{102}-\mathrm{C}_{52} \mathrm{H}_{106}$ ) it may be expected that the distance between kinks would be large, and the flux calculated would have relevance to the crystal growth rates.

What it is necessary to know for the calculation in this paper are the rate constants for the addition and subtraction of a given species in the mixture to and from the growing strip. It is possible by equilibrium considerations to evaluate the free energy change in the system and relate this change to ratios of the rate constants for addition and subtraction, but it is not possible in general to evaluate the rate constants themselves. However, in this problem, various apportionments of this free energy change to the addition and subtraction rate constants have only minor effects on the results.

In the process of carrying out these calculations, specific account had to be taken of the two-dimensional nature of crystallization. This necessitated adapting the LDP theory, which is an exact theory for one-dimensional kinetic problems, to this twodimensional problem. This was accomplished in an approximate, but in our view adequate, manner by appropriate modification of the rate constants.

Although these calculations were carried out on the rather simple binary system $\mathrm{C}_{24} \mathrm{H}_{50}$ and $\mathrm{C}_{26} \mathrm{H}_{54}$, there is in principle no reason why it could not be carried out on more complex mixtures, provided only that the interaction energy in the solid is not so great as to cause eutectic formation. Indeed, in the following paper we apply the LDP method to a system where as many as 50 components must be considered.

\section{Appendix}

In this appendix we derive expressions for $\left(\alpha^{i j} / \beta^{i j}\right)$ that are suitable for our model and our treatment.
The physical model and the concepts are emphasized, while many mathematical steps are omitted.

We calculate these ratios from an ensemble that consists of a "mother liquor" formed by two hydrocarbon components that surrounds a large number of crystals. The crystals present $N_{T}$ substrates to the "mother liquor" upon which strips can be formed. The length of a strip (the number of molecules crystallized from the "mother liquor") is designated by $\nu$ and $\nu$ can take on the values $0,1,2, \ldots, \nu_{0}$.

We assume that all substrates have a uniform thickness, $l_{s}$. This uniform thickness is taken as the average thickness of the substrate, and is given by eq (23) of the text. The assumption of uniform thickness is necessary in order that we can apply our two component one-dimensional rate theory to the problem. This assumption is clearly an approximation, because the substrates will be of uneven rather than of uniform height. We shall see below that this assumption causes a modification of the rate constants.

The strips will be deposited upon the substrates in such a way that the free energy of the ensemble will be minimized. We shall determine the occupation numbers that characterize the strips such that the total free energy is minimized. These occupation numbers can be used to evaluate the ratio $\left(\alpha^{i j} / \beta^{i j}\right)$ by the principle of detailed balance. This is analogous to the method used in single component rate theory. In the present case this leads to [1]

$$
\frac{\alpha^{i j}}{\beta^{i j}}=\left(\frac{P_{\nu+1}^{i j}}{N_{\nu}^{i}}\right)_{e} .
$$

In the present instance the ratio $\left(\alpha^{i j} / \beta^{i j}\right)$ is independent of $\nu$, and we have anticipated this result in writing eq (lA). It should be noted that the occupation numbers on the right-hand side of eq (1A) are not the steady state occupation numbers obtained elsewhere in this paper. The total free energy of our ensemble is

$$
G_{T}=\sum_{\nu=1}^{\nu_{0}} N_{\nu} G_{\nu}+k T \sum_{\nu=0}^{\nu_{0}} N_{\nu} \ln \frac{N_{\nu}}{N_{T}} .
$$

The reference state for this free energy expression is the state where no strips are deposited on the substrates. The average free energy of a strip of length $\nu$ is given by

$$
\begin{aligned}
G_{\nu}=\sum_{j=1}^{2} q_{\nu, 1}^{j}, & {\left[\epsilon_{j}^{0}-\mu_{j}+k T \cdot \ln q_{\nu, 1}^{j}\right] } \\
& +\sum_{\eta=2}^{\nu} \sum_{i=1}^{2} \sum_{j=1}^{2} q_{\nu, \eta}^{i j}\left[\epsilon_{i j}-\mu+k T \ln p_{\nu, \eta}^{i j}\right] .
\end{aligned}
$$

The quantities appearing in eqs (2A) and (3A) have all been defined in the text. The quantities appearing 
in these equations are not independent but must satisfy the following relations:

$$
\begin{gathered}
\sum_{\nu=0}^{\nu_{0}} N_{\nu}=N_{T}, \\
\sum_{j=1}^{2} q_{\nu, 1}^{j}=1 \sum_{i=1}^{2} \sum_{j=1}^{2} q_{\nu, \eta}^{i j}=1, \\
q_{\nu, 1}^{j}=\sum_{i=1}^{2} q_{\nu, 2}^{j i} q_{\nu, \eta}^{j}=\sum_{i=1}^{2} q_{\nu, \eta+1}^{i j}=\sum_{i=1}^{2} q_{\nu, \eta}^{i j} .
\end{gathered}
$$

In addition the conditional probabilities in Equation (3A) satisfy the relations

$$
\begin{gathered}
q_{\nu, \eta}^{i j}=q_{\nu, \eta}^{i} p_{\nu, \eta}^{i j} \\
\sum_{j=1}^{2} p_{\nu, \eta}^{i j}=1 .
\end{gathered}
$$

The fraction of component one in the strips is $\omega$ and is given by

$$
\omega=\frac{\sum_{\nu=1}^{\nu_{0}} N_{\nu} \sum_{\eta=1}^{\nu} q_{\nu, \eta}^{1}}{\sum_{\nu=1}^{\nu_{0}} \nu N_{\nu}} .
$$

We discuss below the relationship between $f_{s}$ and $\omega$ the fractions of component one in the substrate and strips respectively.

We have written down the total free energy of the ensemble. In order to determine the occupation numbers that minimize this free energy, we calculate the variation of $G_{T}$, allowing the $N_{\nu}, q_{\nu, 1}^{j}, q_{\nu, \eta}^{i j}, f_{s}$, and $\omega$ to vary, and equate $\delta G_{T}$ to zero. Since these quantities are not all independent, but instead are related by eq $(4 \mathrm{~A}),(5 \mathrm{~A}),(6 \mathrm{~A})$, and $(9 \mathrm{~A})$, we introduce Lagrange multipliers with these equations. Then the coefficients of $\delta N_{\nu}, \delta q_{\nu, 1}^{j}$, and $\delta q_{\nu, \eta}^{i j}$ are equated to zero.

This leads to quite complicated expressions, but the algebra is relatively straightforward.

The procedure followed here is similar to that employed by Mullins [14] for Markov chains, except that in our case the chains have finite lengths and the matrices involved are not symmetric. We shall not write down the equations involved but merely write down the solutions which are most easily expressed in matrix notation. Let us define the $W$ matrix

$$
\begin{aligned}
(W)_{i j}=w_{i j}= & x_{j} \exp \left\{\frac{\Delta g_{j}}{k T}-\frac{2 a \sigma U\left(l_{j}-l_{i}\right)}{k T}\right. \\
& \left.-\frac{4 a \sigma\left(l_{1}-l_{22}\right)}{k T}\left[\left(1-f_{s}-\Gamma\right) \delta_{i}+\Gamma \omega\right]\right\}
\end{aligned}
$$

The quantity $\Gamma$ in this equation is a Lagrange multiplier associated with the variation of eq (9A).

We also define the row vector

$$
w_{0}^{\prime}=\left(w_{0}^{1}, w_{0}^{2}\right)
$$

where

$$
\begin{aligned}
w_{0}^{j}=x_{j} \exp & \left\{\frac{\Delta g_{j}}{k T}-\frac{2 a \sigma l_{j}}{k T}\right. \\
& \left.-\frac{4 a \sigma\left(l_{1}-l_{2}\right)}{k T}\left[\left(1-f_{s}-\Gamma\right) \delta_{1}^{j}+\Gamma \omega\right]\right\}
\end{aligned}
$$

With these quantities we can write down our solutions as

$$
\begin{aligned}
q_{\nu, \eta}^{i j}= & \frac{w_{0}^{\prime}(W)^{\mu-2}\left(\begin{array}{l}
\delta_{1}^{i} \\
\delta_{2}^{i}
\end{array}\right) \omega_{i j}\left(\delta_{1}^{j}, \delta_{\underline{j}}^{j}\right)(W)^{\nu-\eta_{1}}\left(\begin{array}{l}
1 \\
1
\end{array}\right)}{w_{0}^{\prime}(W)^{\nu-1}\left(\begin{array}{l}
1 \\
1
\end{array}\right)} \\
q_{\nu, \eta}^{j}= & \frac{w_{0}^{\prime}(W)^{\eta-1}\left(\begin{array}{l}
\delta_{j}^{j} \\
\delta_{i}
\end{array}\right)\left(\delta_{i}^{j}, \delta_{\underline{1}}^{j}\right)(W)^{\nu-\eta}\left(\begin{array}{l}
1 \\
1
\end{array}\right)}{w_{0}^{\prime}(W)^{\nu-1}\left(\begin{array}{l}
1 \\
1
\end{array}\right)}
\end{aligned}
$$

and

$$
\frac{N_{\nu}}{N_{0}}=w_{0}^{\prime}(W)^{\nu-1}\left(\begin{array}{l}
1 \\
1
\end{array}\right)
$$

These solutions allow us to evaluate the occupation numbers and evaluate the ratio of the rate constants.

$$
\frac{\alpha^{i j}}{\beta^{i j}}=\left(\frac{P_{\nu+1}^{i j}}{N_{\nu}^{i}}\right)_{e}=\frac{N_{\nu+1} q_{\nu+1, \nu+1}^{i j}}{N_{\nu} q_{\nu, \nu}^{i}}=w_{i j}
$$

We therefore have an expression for the ratio of the rate constants.

Our solution is not complete, for the elements of the $(W)$ matrix contain the quantities $\Gamma$ and $\omega$, which as yet have not been determined. We can obtain two further relationships. From eqs (9A), (14A), and (15A) it can be shown that for $\nu_{0}$ very large

$$
f=\frac{z-w_{22}}{2 z-w_{11}-w_{22}}
$$

where $z$ is the largest eigenvalue of the $W$ matrix. This equation provides a relationship between $\omega$ and $\Gamma$.

A further relation is obtained by considering the variation of the ensemble total free energy. Using Equations (13A), (14A), and (15A) this becomes

$$
\delta G_{T}=4 a \sigma\left(\sum_{\nu=1}^{\nu_{0}} \nu N_{\nu}\right)\left(\Gamma \delta \omega-\omega \delta f_{s}\right)
$$

This equation relates the variation of the total free 
energy of the ensemble to the variation in the composition of the substrates and strips.

The two dimensional character of our problem must be kept in mind in our treatment of this equation. This can be illustrated by considering a truly one dimensional problem as an example: Let the mother liquor and strips be the two component system of hydrocarbons under consideration, but suppose the substrate consists of a third component, say still another hydrocarbon. Then we need consider no variation in $f_{s}$ i.e., $\delta f_{s}=0$. In this case in order that $\delta G_{T}=0$, we set $\Gamma=0$. The ratios of the rate constants become the usual Boltzmann factors that were derived in [1]. These rate constants are the proper ones for the one dimensional problem. However, if we follow this procedure in the present problem (i. e., set $\left.\delta F_{s}=0, \Gamma=0\right)$, the resulting rate constants are not the proper ones. This is most clearly seen when the equilibrium phase diagram has minima in the liquidus and solidus curves. These minima do not occur at the same value of the concentration, which would imply a region of concentrations where no stable equilibrium exists.

We must therefore treat eq (18A) more carefully. Suppose we have a particular liquid concentration at a particular temperature. There will exist a crystal concentration, $f_{e}\left(x_{1}, T\right)$, that is most stable. That is, if we vary the concentration of the crystal $\left(\delta f=\delta f_{s}\right)$

$$
\delta G_{T}=4 a \sigma\left(\sum_{\nu=1}^{\nu 0} \nu N_{\nu}\right)\left(\Gamma-f_{e}\right) \delta f_{s}=0
$$

so that

$$
\Gamma=f_{e}\left(x_{1}, T\right)=\omega=f_{s} .
$$

This property must hold at equilibrium (i.e., where $x_{1}, T$ fall on the equilibrium liquidus curve), but it also will hold at other concentrations and temperatures. This result does not solve our problem, however, except at particular values. We wish a value of $\Gamma$ that holds for any values of $\left(x_{1}, T, f_{s}\right)$, while eq (19A) yields a value of $\Gamma$ that holds for $\left(x, T, f_{s}=f_{e}\right)$. When the kinetics of the crystallization process leads to values of $f_{s} \neq f_{e}$, it cannot be expected that $\delta G_{T}$ vanishes.

A more critical analysis would be required to obtain values of $\Gamma$ for general values of $\left(x_{1}, T, f_{s}\right)$. Such an analysis does not seem worthwhile, because we have already introduced the approximation that the substrate height is uniform. Instead we shall assume

$$
\Gamma=f_{s} .
$$

This assumption satisfies eq (19A) when $f_{s}=f_{e}$, and should be reasonably accurate at other values of $f_{s}$. At thermal equilibrium it satisfies our requirements.

We therefore have

$$
\begin{aligned}
\frac{\alpha^{i j}}{\beta^{i j}}=x_{j} \exp \{ & \frac{\Delta g_{j}}{k T}-\frac{2 a \sigma U\left(l_{j}-l_{i}\right)}{k T} \\
& \left.-\frac{4 a \sigma\left(l_{1}-l_{2}\right)}{k T}\left[\left(1-2 f_{s}\right) \delta_{1}^{j}+f_{s} \omega\right]\right\} .
\end{aligned}
$$

In this equation $\omega$ is the value that must satisfy eq (17A). This can be expressed as

$$
2 \omega-1=\frac{\xi-1+x_{1}}{\sqrt{\left(\xi-1+x_{1}\right)^{2}+4\left(1-x_{1}\right) \xi \exp \left\{\frac{2 a \sigma\left(l_{1}-l_{2}\right)}{k T}\right\}}}
$$

where

$$
\xi=x_{1} \exp \left\{\frac{\Delta g_{1}}{k T}-\frac{\Delta g_{2}}{k T}-\frac{4 a \sigma\left(l_{1}-l_{2}\right)\left(1-2 f_{s}\right)}{k T}\right\} .
$$

Then we see that for any values of $\left(x_{1}, T, f_{s}\right)$ we can obtain $\omega$ from eqs $(22 \mathrm{~A})$ and $(23 \mathrm{~A})$. Then the ratios $\left(\alpha^{i j} / \beta^{i j}\right)$ are obtained from eq (21A). Equation (21A) is the same as eq (28) of the text.

\section{References}

[1] J. I. Lauritzen, Jr., E. A. DiMarzio and E. Passaglia, J. Chem. Phys. 45, 4444 (1966).

[2] J. I. Lauritzen, Jr. and J. D. Hoffman, J. Res. NBS 64A (Phys. and Chem.) No. 1, 73 (1960): J. D. Hoffman and J. I. Lauritzen, Jr., Ibid. 65A (Phys. and Chem.) No. 2, 297 (1960).

[3] F. C. Frank and M. Tosi, Proc. Roy. Soc. A263, 323 (1961).

[4] F. P. Price, J. Chem. Phys. 35, 1884 (1961).

[5] W. K. Burton, N. Cabrera and F. C. Frank, Phil. Trans. Roy Soc. (London) A243, 299 (1951).

[6] I. M. Dawson and V. Vand, Proc. Roy. Soc. A206, 555 (1951).

[7] W. M. Mazee, Anal. Chim. Acta 17,97 (1951).

[8] W. M. Mazee, Erdöl u. Kohle 13, 88 (1960).

[9] D. Turnbull and R. L. Cormia, J. Chem. Phys. 34, 820 (1961).

[10] M. G. Broadhurst, J. Research NBS 66A (Phys. and Chem.) No. 3, 241 (1962).

[11] P. J. Flory, R. A. Orwall and A. Vriz, J. Phys. Chem. 86, 3515 (1964).

[12] V. Vand, Acta. Cryst. 6, 797 (1953).

[13] J. C. Slater, Introduction to Chemical Physics, (McGrawHill Book Co., New York, N.Y., 1939).

[14] W. W. Mullins, Phys. Rev. 1 14, 389 (1959).

(Paper 71A4-457) 